\title{
Mixed-State Transport Characteristics of Magnesium Diboride Films
}

\author{
M. N. Kunchur, Cheng Wu, D. H. Arcos, G. Saracila, \\ Department of Physics and Astronomy, University of South Carolina, Columbia, SC 29208, U.S.A. \\ Eun-Mi Choi, Kijoon H.P. Kim, W. N. Kang, and Sung-Ik Lee \\ National Creative Research Initiative Center for Superconductivity and Department of Physics \\ Pohang University of Science and Technology, Pohang 790-784, Republic of Korea
}

Received on 23 May, 2003.

\begin{abstract}
We have investigated the low-temperature $\left(T<T_{c} / 10\right)$ mixed-state current-voltage (IV) response of magnesium diboride films beyond the point where the superconductivity is completely destroyed and the system enters the normal state. The resistance-versus-current $R(I)$ curves are extremely steep and featureless, with a critical current density $j_{c}$, marking the onset of dissipation, that is unusually high $\left(j_{c}>j_{d} / 10\right)$ with respect to the depairing current density $j_{d}$. At large flux densities $H_{c 2} / 10 \lesssim B \lesssim H_{c 2}$, the $R(I)$ curve has a functional shape that is largely independent of $B$, indicating that the rise in resistivity with increasing current occurs mainly due to pair-breaking rather than flux motion. The macroscopic destruction current $I^{*}$, which drives the system normal, has a $\left[1-\sqrt{B / H_{c 2}}\right]$ flux-density dependence, suggesting that the vortices mainly reduce the effective cross section over which a current of effective density $j \sim j_{d}$ flows.
\end{abstract}

\section{Introduction and background}

When a superconducting state is formed, charge carriers correlate and condense into a coherent macroscopic quantum state. The formation of this state is governed principally by a competition between four energies: condensation, magnetic-field expulsion, thermal, and kinetic. The order parameter $\Delta$, that describes the extent of condensation and the strength of the superconducting state, is reduced as the temperature $T$, magnetic field $H$, and electric current density $j$ are increased. The boundary in the $T-H-j$ phase space that separates the superconducting and normal states is where $\Delta$ vanishes, and the three parameters attain their critical values $T_{c}(H, j), H_{c 2}(T, j)$, and $j_{d}(T, H) . j_{d}$ is the depairing or pair-breaking current density, which sets the intrinsic upper limiting scale for current transport in any superconductor.

In a transport measurement in finite flux density $B$, resistance appears above a threshold $j_{c}$ at which flux vortices start to move. In a system with weak flux pinning, $j_{c}$ is much lower than $j_{d}$ and the response goes through alternate regimes of Ohmic (homogeneously linear dependence of $V$ on $I$ ) and non-Ohmic behavior [1]. At very low driving forces (low $j$ ) there can be observable resistance due to thermally activated flux flow (TAFF) or flux creep. Then one encounters a non-linear response as current driven depinning sets in; in effect the number of mobile vortices is rising with $j$. This is incipient flux flow. At sufficiently larger $j$, the vortex motion is effectively free from the influence of pinning and the response becomes Ohmic again. We previously introduced the term free flux flow (FFF) for this linear regime [2]. Here the dissipation and resistivity should correspond to the canonical $\rho_{f} \sim \rho_{n} B / H_{c 2}$ Bardeen-Stephen expression (sometimes large departures can occur for exceptional situations such as superclean systems and narrow vortex cores where the internal energy-level spacing exceeds their widths). Beyond FFF, the response can become non-linear because of the heating of the electron gas [3] or changes in the electron distribution function [4]. Finally at yet higher currents, pair-breaking destroys superconductivity and drives the system normal and the resistance again becomes independent of current, being characteristic of the normal state. Our previous review article discusses some of these stages of dissipation. In $\mathrm{Y}_{1} \mathrm{Ba}_{2} \mathrm{Cu}_{3} \mathrm{O}_{7}$, the depinning critical current is sufficiently weak compared to the pairbreaking value that all of these regimes have be observed.

In $\mathrm{MgB}_{2}$ the scenario has turned out to be completey different from the cuprates. The pinning in $\mathrm{MgB}_{2}$ films is much stronger (because of its higher isotropy and ten times larger vortex cross section) and its $T_{c}$ is intermediate in value (less than half that of $\mathrm{Y}_{1} \mathrm{Ba}_{2} \mathrm{Cu}_{3} \mathrm{O}_{7}$ or $\mathrm{Bi}_{2} \mathrm{Sr}_{2} \mathrm{CaCu}_{2} \mathrm{O}_{8}$ ). This boosts $j_{c}$ while inhibiting thermally activated flux motion. On the other hand $j_{d}$ has a much lower value than cuprates $\left(j_{d}(0) \sim 10^{7} \mathrm{~A} / \mathrm{cm}^{2}[5]\right)$. As a result $j_{c}$ is within an order of magnitude of $j_{d}$ and the IV curve remains dissipationless until $j_{c} \sim j_{d}$ and then quickly rises to the normal-state 
resistance more because of pair-breaking than flux motion.

Another distinction between $\mathrm{MgB}_{2}$ and cuprates is its much lower normal-state resistivity $\rho_{n}$ (lower than cuprates by 1-2 orders of magnitude). This leads to a much stiffer viscous coefficient $\eta\left(\approx \Phi_{0} H_{c 2} / \rho_{n}\right)$ and consequently much lower vortex velocities for comparable values of applied $j$. Hence non-equilibrium effects related to changes in the macroscopic electronic distribution function [3, 4] are suppressed. All of these factors combine to produce an extremely steep non-linear IV curve influenced only secondarily by the motion of flux vortices, even when $B$ approaches $H_{c 2}$.

\section{Experimental details}

The samples are $400 \mathrm{~nm}$ thick films of $\mathrm{MgB}_{2}$ fabricated using a two-step method whose details are described elsewhere[6, 7]. An amorphous boron film was deposited on a (1 $1 \overline{1} 02) \mathrm{Al}_{2} \mathrm{O}_{3}$ substrate at room temperature by pulsedlaser ablation. The boron film was then put into a $\mathrm{Nb}$ tube with high-purity $\mathrm{Mg}$ metal (99.9\%) and the $\mathrm{Nb}$ tube was then sealed using an arc furnace in an argon atmosphere. Finally, the heat treatment was carried out at $900^{\circ} \mathrm{C}$ for $30 \mathrm{~min}$. in an evacuated quartz ampoule sealed under high vacuum. X-ray diffraction indicates a highly c-axis-oriented crystal structure normal to the substrate surface with no impurity phases. The films were photolithographically patterned down to narrow bridges. In this paper we show data on three bridges, labelled S, M, and L (for small, medium, and large) with lateral dimensions $2.8 \times 33,3.0 \times 61$, and $9.7 \times 172 \mu \mathrm{m}^{2}$ respectively. The lateral dimensions are uncertain by $\pm 0.7 \mu \mathrm{m}$ and the thickness by $\pm 50 \mathrm{~nm}$. Fig. 1(a) shows the sample geometry. The horizontal sections of the current leads contribute a small $(\sim 15 \%)$ series resistance to the resistance of the actual bridge when $B \gtrsim H_{c 2}(T)$ and the entire material is normal. At low $B$ in the mixed state, this extra contribution is frozen out because the current is spread out in these wider lead areas and hence its density is too low to cause dissipation. Then $j$ is high only within the bridge itself and the leads do not contribute to the measured resistance.

The non-linear electrical transport measurements were made using a pulsed signal source with pulse durations ranging from 0.1 to $4 \mu \mathrm{s}$ and a duty cycle of about $1 \mathrm{ppm}$. A conventional continuous DC method was employed for the very low currents ( $I=1.4 \mu \mathrm{A})$. Fig. 1(b) shows pulse waveforms under the especially severe conditions of $j=9.7 \mathrm{MA} / \mathrm{cm}^{2}$, $E=83 \mathrm{~V} / \mathrm{cm}$, and $p=j E=803 \mathrm{MW} / \mathrm{cm}^{3}$. The resistance rises to $90 \%$ of its final value in about $50 \mathrm{~ns}$ from the $10 \%$ onset of $I$. From a knowledge of the thermal conductivities and specific heat capacities of the film and substrate materials, and their mutual thermal boundary resistance, one can calculate the total thermal resistance $R_{t h}$ for any pulse duration [1,8]. Also if $R(T)$ has enough variation, the film's
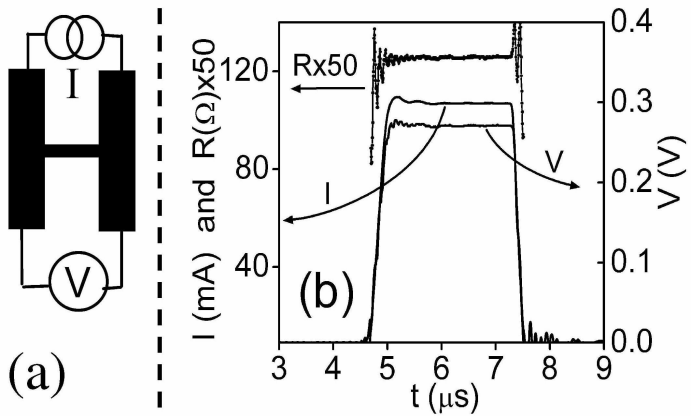

Figure 1. (a) Sample geometry used for resistance measurement. At low values of $j$ the wide lead areas add a constant resistance of about $15 \%$ of the total value. At high $j$ this contribution is frozen out. (b) Pulse waveforms under worst-case conditions $(j=9.7$ $\mathrm{MA} / \mathrm{cm}^{2}, E=83 \mathrm{~V} / \mathrm{cm}$, and $p=j E=803 \mathrm{MW} / \mathrm{cm}^{3}$ on the plateaus). The resistance rises to (90\% of) its final value in about $50 \mathrm{~ns}$ from the $(10 \%)$ onset of $I$.

own resistance can be used as a thermometer to measure $R_{t h}$. For films of $\mathrm{Y}_{1} \mathrm{Ba}_{2} \mathrm{Cu}_{3} \mathrm{O}_{7}$ (YBCO) on $\mathrm{LaAlO}_{3}$, which were used for most of our previous work, we found $R_{t h} \sim$ $1-10 \mathrm{nK} . \mathrm{cm}^{3} / \mathrm{W}$ at microsecond timescales $[3,1,9]$. In the case of our $\mathrm{MgB}_{2}$ films, we expect $R_{t h}$ to be smaller because of sapphire's very high conductivity. However the five parameters required to calculate $R_{t h}$ from first principles are not all known for this film-substrate combination and $\mathrm{MgB}_{2}$ has a very flat $R(T)$ below $50 \mathrm{~K}$, so one can't measure $R_{t h}$ as was done for YBCO. We can, however, obtain an upper bound on $R_{t h}$ in the following way: Fig. 2 shows $I V$ curves for sample $\mathrm{L}$ in zero field (This is the largest sample with the lowest surface-to-volume ratio, so that it represents the worst case thermal scenario.). The curves were measured with the sample in different thermal environments. Above some threshold current $I_{d} \sim 650 \mathrm{~mA}$, the system abruptly switches into the normal state. The value of $I_{d}$ is not sensitive to the thermal environment contacting the exposed surface of the film, confirming that the highly conductive sapphire, together with the greatly reduced heat input during the short pulse, prevents the film's temperature from rising significantly (It has been shown by Stoll et al. [10] that if there is sample heating, the thermal environment makes a significant difference because it will provide an additional path for the heat to flow through.). We show elsewhere [5] that this jump to the normal state occurs due to pairbreaking by the current ${ }^{1}$. At the point the system is driven normal, the power density reaches $p=j E=1.01 \mathrm{GW} / \mathrm{cm}^{3}$. This sets a gross upperbound of $R_{t h} \sim 7 \mathrm{nK} . \mathrm{cm}^{3} / \mathrm{W}$. Note that the main bottle neck of heat conduction is the film-substrate boundary resistance which is not strongly temperature dependent [8]. In the present work, typical $p$ values are two orders of magnitude lower than the critical $1 \mathrm{GW} / \mathrm{cm}^{3}$ and so we expect the temperature rise to be a small fraction $(\sim 1$ $\%$ ) of $T_{c}$. A value of $j_{d}(0) \approx 2 \times 10^{7} \mathrm{~A} / \mathrm{cms}$ was obtained

\footnotetext{
${ }^{1}$ The value of the current density at which this jump occurs, $\sim 2 \times 10^{7} \mathrm{~A} / \mathrm{cm}^{2}$, is roughly comparable to the theoretical estimate of $j_{d}(0)=$ $c H_{c}(0) /[3 \sqrt{6} \pi \lambda(0)] \sim 6 \times 10^{7} \mathrm{~A} / \mathrm{cm}^{2}$.
} 
from such zero-field IV curves [5]. The corresponding values for the zero-field pair-breaking currents are $240 \mathrm{~mA}, 257$ $\mathrm{mA}$, and $700 \mathrm{~mA}$ for samples S, M, and L respectively [5].

The magnetic field is applied normal to the film (parallel to the $c$ axis) and the self field of the current is much lower than the applied fields used in this work. Further details of the measurement techniques have been published in a previous review article [1] and other recent papers [9, 3].

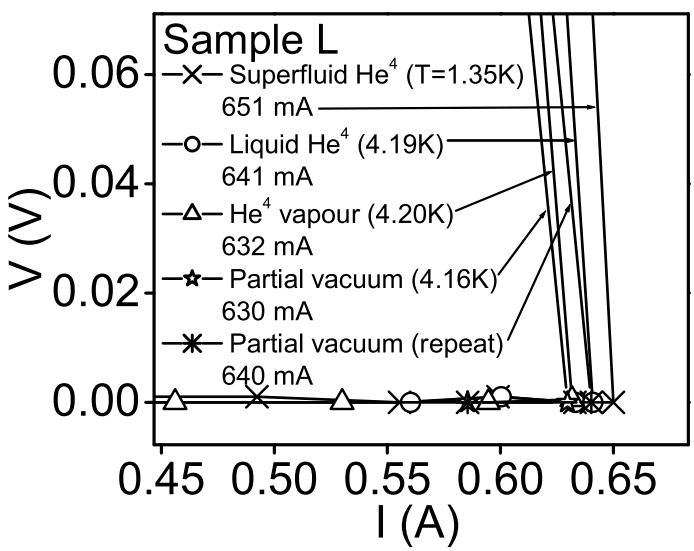

Figure 2. IV curves in zero field for sample $L$ in different thermal environments. The resistance is driven to its normal-state value (top parts of curves lie outside the panel) by currents of pairbreaking magnitudes. The absence of a significant systematic dependence on the thermal environment shows the paucity of sample heating.

\section{Results and analysis}

Fig. 3(a) shows the $\mathrm{R}(\mathrm{I})$ curves for sample $\mathrm{M}$ at different values of $B$ in the $H_{c 2} / 10 \lesssim B \lesssim H_{c 2}$ range. After the onset of dissipation, the resistance quickly rises to the full normalstate value. It should be noted that the plateaus do not correspond to FFF but to the normal state. Accordingly the resistance value changes very little with the applied $B$ (the slight shift in plateau resistance at the highest flux densities can be understood in terms of spreading of resistance outside the bridge area into the current-lead areas as explained in the experimental section; fields approaching $H_{c 2}$ start driving the whole film normal so that the resistance of the wider current-lead areas is not frozen out). It is interesting to note that the overall shapes of the curves are almost independent of $B$. The curves of panel (a) can be made to overlap, as shown in Fig. 3(b), by merely shifting them vertically and horizontally by constant amounts. For a dissipation curve influenced by flux motion, one expects the function to depend on $B$. The vertical shift is not crucial as explained above. The horizontal shift corresponds to substracting an amount $I^{*}$ from $I$. $I^{*}$, which we call the destruction current, is the elbow point (arrow in Fig. 3(b)) below which $R$ starts to fall below its normal-state value. In panel (c), this $I^{*}$ is plotted versus the function $\left[1-\sqrt{B / H_{c 2}}\right]$, the meaning of which is explained below. It is seen that most of the data obey a roughly linear dependence that extrapolates back to the origin, consistent with the pinch-off model. Also we note that at the current scale of this phenomenon $I^{*} \sim 50 \mathrm{~mA}$ is of the order of the pair-breaking current $(257 \mathrm{~mA}$ ) for this sample (For $B<2 \mathrm{~T}$, the sample resistance becomes lower than the source impedance of the pulsed voltage source. As a result the relevant portion of the $R(I)$ curve is inaccessible. This external circuit issue is discussed elsewhere [5]).
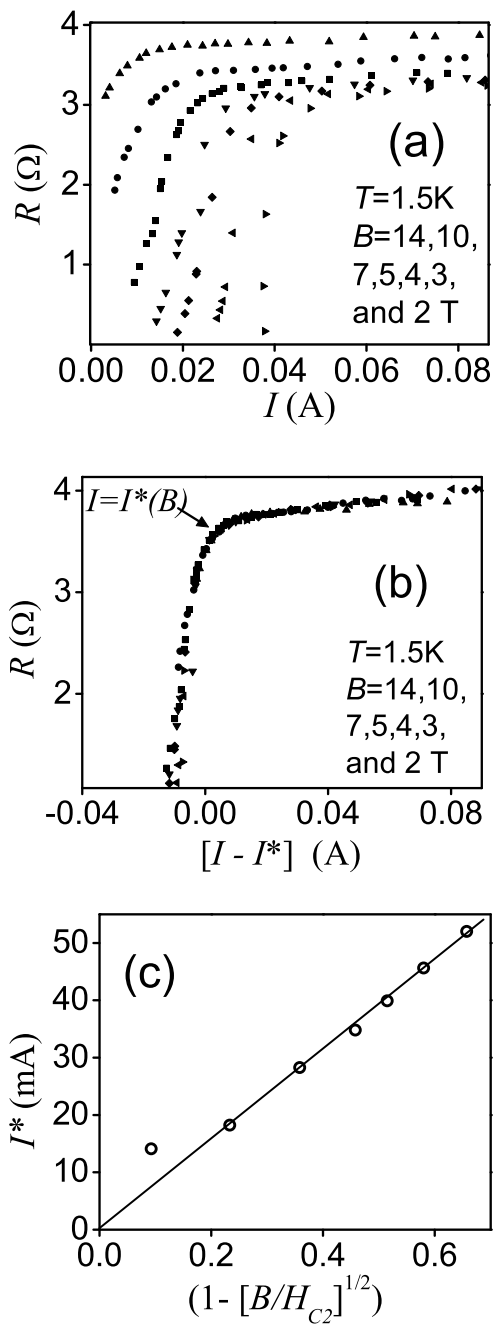

Figure 3. (a) Resistance versus current curves for sample M. Flux densities are indicated from left to right. (b) The same data now plotted with simple vertical and horizontal shifts to produce a single collapsed curve. The horizontal shifts are by the amount $I^{*}$, the destruction current, defined at the elbow of the curve as indicated by the arrow. (c) Plot of the destruction current versus the pinch-off function $\left(1-\sqrt{B / H_{c 2}}\right)$.

We tentatively propose the following explanation for the $R(I)$ curve and its parallel shifts with $B$ : Clearly the current scale for the curves is of the order of the pair-breaking current (please earlier discussion and reference [5]). This implies that pinning is so strong that vortex motion doesn't lead to enough dissipation to greatly contribute to $R(I)$. Then the perfect conductivity is destroyed mainly by pairbreaking and the field merely serves to suppress the effective $j_{d}$. We could not find in the literature any discussion of the field dependence of $j_{d}$. For the field range in question we propose that the main effect of increasing $B$ is to 
"pinch off" the cross section by adding more vortices. At high $B \sim H_{c 2}$, there will be some additional corrections as the field approaches its critical value, but for lower fields let's assume that the local $j_{d}$ is unaffected by $B$, but only the macroscopic average $j_{d}$ is reduced because of loss of crosssectional area. In this case the destruction current $I^{*}$ will equal $j_{d}$ times the available cross section which is given by the factor $\left[1-\sqrt{B / H_{c 2}}\right]$.
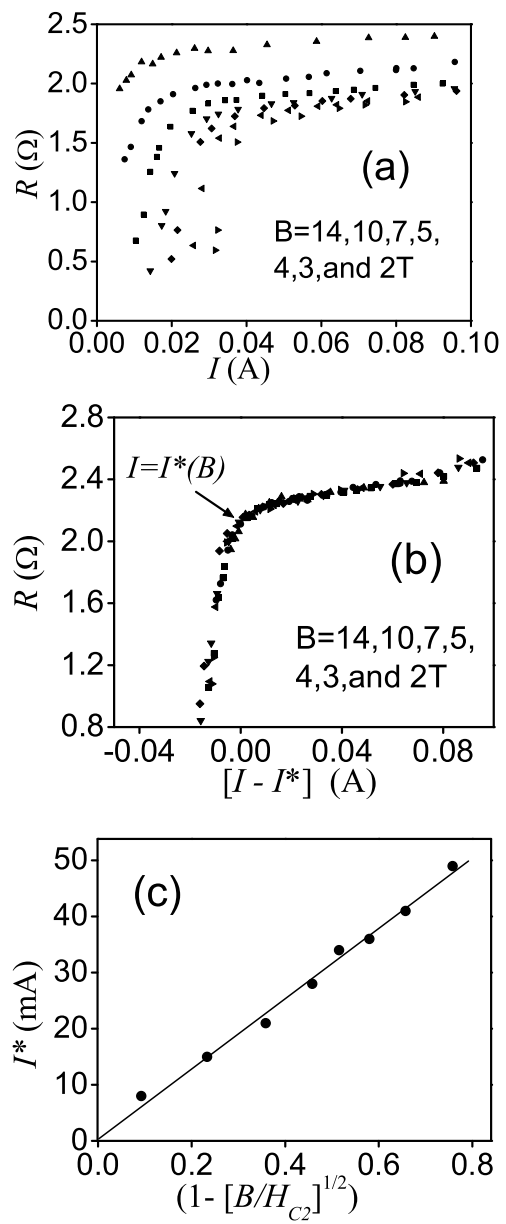

Figure 4. Data plots for sample S. (a) Resistance versus current curves. Flux densities are indicated from left to right. (b) The collapsed data plotted after simple vertical and horizontal shifts. (c) The destruction current (defined at the elbow of the $R(I)$ curve in above panel) plotted versus the pinch-off function $\left[1-\sqrt{B / H_{c 2}}\right]$.

Fig. 4 shows a similar set of curves for sample $\mathrm{S}$ (sample $\mathrm{L}$ also looks similar). In all cases, the $R(I)$ curves at different flux densities collapse when shifted. And $I^{*}$, which amounts to the horizontal shift along the current axis, is proportional to the pinch-off function consistent with our simple model of vortices choking of the current-carrying cross section (other effects like pair-breaking by the field and rise in $R$ due to vortex motion seem to be secondary).

\section{Summary}

We have investigated the low-temperature $\left(T \ll T_{c}\right)$ in-field transport behavior of $\mathrm{MgB}_{2}$ and present the first measurement of the full dissipation curves (i.e., $0 \lesssim j \lesssim j_{d}$ and $0 \leq R(T=0) \lesssim R_{n}$ ) for this system. $\mathrm{MgB}_{2}$ films made by the two-step laser-ablation process have intrinsically strong pinning, leading to ususually steep IV and $R(I)$ curves. The onset of dissipation is within an order of magnitude of the pairbreaking current, even at flux densities of a few Teslas, and the resistance quickly rises to the full normal-state value as the current is increased beyond $j_{c}$. The dissipation curve seems to be controlled mainly by the pair-breaking action of the current, the vortices merely serving to reduce the effective cross section over which the transport current is able to flow. Such a "pinch-off" model provides a first-order explanation of the relative horizontal (current-axis) displacements of the $R(I)$ curves at different flux densities.

\section{Acknowledgements}

The authors acknowledge useful discussions with J. M. Knight and B. I. Ivlev. This work was supported by the U. S. Department of Energy through grant number DE-FG0299ER45763 and by the Ministry of Science and Technology of Korea through the Creative Research Initiative Program.

\section{References}

[1] M. N. Kunchur, Mod. Phys. Lett. B. 9, 399 (1995).

[2] M. N. Kunchur, D. K. Christen, and J. M. Phillips, Phys. Rev. Lett. 70, 998 (1993).

[3] M. N. Kunchur, Phys. Rev. Lett. 89, 137005 (2002).

[4] A. I. Larkin and Yu. N. Ovchinnikov, Zh. Eksp. Teor. Fiz. 68, 1915 (1975) [Sov. Phys. JETP 41, 960 (1976)].

[5] M. N. Kunchur et al., Phys. Rev. B, 68, 064516 (2003).

[6] W. N. Kang et al., Science 292, 1521 (2001).

[7] W. N. Kang et al., Physica C 385, 24 (2003).

[8] S. K. Gupta et. al., Physica C 206, 335 (1993) and references therein; and M. Nahum et. al., Appl. Phys. Lett. 59, 2034 (1991) and references therein.

[9] M. N. Kunchur, B. I. Ivlev, D. K. Christen, J. M. Phillips, Phys. Rev. Lett. 84, 5204 (2000).

[10] O. M. Stoll, S. Kaiser, R. P. Huebener, and M. Naito, Phys. Rev. Lett. 81, 2994 (2001). 